\title{
Cardio-Vascular Effects in Hypothyroidism - A Cross-Sectional Study
}

\author{
V Gurudatta Murthy \\ Assistant Professor, Department of Medicine, Sapthagiri Institute of Medical Sciences and Research Institute, Bengaluru, India
}

Corresponding author: Dr V Gurudatta Murthy, Assistant Professor, Postal Address- No-360, 1st Floor, 11th Cross, MPM Layout, Mallathalli Main Road (Near Malathalli Lake), Bengaluru-560026, India

DOI: http://dx.doi.org/10.21276/ijcmsr.2019.4.1.8

How to cite this article: V Gurudatta Murthy. Cardio-Vascular Effects in Hypothyroidism - A Cross-Sectional Study. International Journal of Contemporary Medicine Surgery and Radiology. 2019;4(1):A31-A34.

\section{A B S T R A C T}

Introduction: The low serum concentrations of thyroid hormones are associated with a decrease in cardiac output, heart rate, stroke volume, and myocardial contractility, and an increase in systemic vascular resistance. Several potential cellular mechanisms by which chronic low thyroid function may contribute to HF have been identified. Hypothyroidism may lead to altered blood lipids and accelerated atherosclerosis, Stimulation of myocardial fibrosis, Vasoconstriction, Induction of a gene program resembling that of pathological hypertrophy. Cuttent study was done with the objective to study various cardiac manifestations in hypo-thyroidism patients

Material and methods: It was a cross sectional study consisting of 54 cases of hypo-thyroidism patients, is undertaken to study the cardiac manifestations by ECG and ECHO at tertiary care hospital from January 2016 to December 2016.

Results: In the present study which included 54 hypothyroid patients, 40 were females and 14 were males. Among cardiovascular symptoms breathlessness was commonest symptom being present in 27 patients followed by palpitation in 17 patients and chest pain in 6 patients. Among ECG changes, sinus bradycardia was commonest being present in 20 patients, followed by Low voltage complexes in 11 patients, ST-T changes in 10 patients, Long QT in 6 patients, Ectopics in 3 patients, Axis deviation in 2 patients, Prolonged PR in 1 patient, RBBB in 1 patient. Diastolic dysfunction seen in 17 patients, pericardial effusion ( 25 cases) being commonest echo-cardiographic changes in hypothyroid patients followed by, Left ventricular hypokinesia in 8 patients, LVH in 4 patients.

Conclusion: The study showcases the significant cardio-vascular changes among the vulnerable age group between 20-40 years. Predominantly females were more affected than males. Hypo-thyrodism patients can have significant cardio-vascular symptoms, breathlessness being the most common symptom followed by palpitation and chest pain. Study of serial ECG changes revealed sinus bradycardia as the commonest finding followed by low voltage complexes, ST-T changes, long QT, ectopics, axis deviation, prolonged PR interval and RBBB.

Keywords: Cardio-Vascular, Hypothyroidism, Sinus Bradycardia, Low Voltage Complex, ECG, Echo-Cardiography.

\section{INTRODUCTION}

The low serum concentrations of thyroid hormones are associated with a decrease in cardiac output, heart rate, stroke volume, and myocardial contractility, and an increase in systemic vascular resistance. ${ }^{1}$

Several potential cellular mechanisms by which chronic low thyroid function may contribute to $\mathrm{HF}$ have been identified. Hypothyroidism may lead to altered blood lipids and accelerated atherosclerosis, Stimulation of myocardial fibrosis, Vasoconstriction, Induction of a gene program resembling that of pathological hypertrophy. ${ }^{2}$

The most consistent cardiac abnormality recognized in patients with overt hypothyroidism is impairment of LV diastolic function, which is characterized by slowed myocardial relaxation and impaired early ventricular filling.32 Hypothyroidism led to severe, progressive systolic dysfunction and increased chamber diameter/wall thickness ratio despite a reduction in cardiac mass. ${ }^{3}$
Both phases of heart cycle are early and contemporary involved in subclinical hypothyroidism causing the decrease of intramyocardial contractility and the impairment of both active and passive phases of diastole. Left ventricular ejection fraction, measured by radio-nucleotide ventriculography, was reduced in hypothyroid patients at rest and on exercise. Hypothyroid cardio-myopathy is an uncommon manifestation of hypothyroidism that can take the form of dilated cardiomyopathy. ${ }^{4}$

Clinical hypothyroidism is associated with premature atherosclerosis and increased prevalence of coronary disease. This is at least partly due to the lipid abnormalities often found in hypothyroidism. Possible mechanisms behind the link between hypothyroidism and atherosclerosis, other than dyslipidemia, include the effects of thyroid hormones on coagulation, vasodilation, parasympathetic function, and homocysteine metabolism. Hypothyroidism is associated with decreased endothelium-dependent vasodilatation, and 


\begin{tabular}{|c|c|c|c|c|c|c|}
\hline \multirow[t]{2}{*}{ Age group (years) } & \multicolumn{2}{|c|}{ Male } & \multicolumn{2}{|c|}{ Female } & \multicolumn{2}{|c|}{ Total } \\
\hline & No. & $\%$ & No. & $\%$ & No. & $\%$ \\
\hline $21-40$ & 8 & 15 & 20 & 37 & 28 & 52 \\
\hline $41-60$ & 1 & 2 & 18 & 33 & 18 & 35 \\
\hline$>60$ & 5 & 9 & 2 & 4 & 8 & 13 \\
\hline Total & 14 & 26 & 40 & 74 & 54 & 100 \\
\hline
\end{tabular}

\begin{tabular}{|l|c|c|c|}
\hline Cardiovascular symptoms & Male & Female & Total \\
\hline Breathlessness & 6 & 21 & 27 \\
\hline Palpitation & 3 & 14 & 17 \\
\hline Chest pain & 1 & 5 & 6 \\
\hline Multiple symptoms & 1 & 3 & 4 \\
\hline \multicolumn{4}{|l|}{ Table-2: Cardiovascular symptoms of hypothyroid patients } \\
\hline
\end{tabular}

\begin{tabular}{|l|c|c|c|}
\hline ECG changes & Male & Female & Total \\
\hline Sinus bradycardia & 8 & 12 & 20 \\
\hline Low voltage complex & 3 & 8 & 11 \\
\hline ST-T changes & 1 & 9 & 10 \\
\hline Long QT & 2 & 4 & 6 \\
\hline Ectopics & 1 & 2 & 3 \\
\hline Axis deviation & 1 & 1 & 2 \\
\hline Prolonged PR & 0 & 1 & 1 \\
\hline RBBB & 1 & 0 & 1 \\
\hline \multicolumn{4}{|l|}{ Table-3: Electrocardiographic changes in hypothyroidism } \\
\hline
\end{tabular}

\begin{tabular}{|l|c|c|c|}
\hline Echo-cardiographic changes & Male & Female & Total \\
\hline Diastolic dysfunction & 4 & 13 & 17 \\
\hline Pericardial effusion & 4 & 21 & 25 \\
\hline Left ventricular hypokinesia & 0 & 8 & 8 \\
\hline LVH & 2 & 2 & 4 \\
\hline \multicolumn{4}{|c|}{ Table-4: Echocardiographic studies in hypothyroidism } \\
\hline
\end{tabular}

animal studies have proposed that the thyroid status alters the capacity for both formation and response to nitric oxide. Endothelial dysfunction in patients with $\mathrm{SCH}$ may result from reduction in nitric oxide availability, with resultant impairment of flow-mediated vasodilatation with resultant impairment of flow-mediated vasodilatation. ${ }^{5}$

Hypothyroidism has important electrophysiological effects, such as prolongation of the $\mathrm{QRS}$ complex and the $\mathrm{QT}$ interval which may predispose to the development of ventricular tachyarrhythmias.16 Electrocardiographic changes includes sinus bradycardia, prolongation of PR interval, low amplitude of $\mathrm{P}$ waves and QRS complex, alteration of ST segments and flattened or inverted $T$ waves. This may be due to pericardial effusion. RBBB, LBBB and rarely complete heart block may present. Singh et al. describes three cases with varying degrees of atrioventricular block in myxedema, which reverted to normal sinus rhythm following thyroid hormone replacement. ${ }^{6}$

Echocardiography shows Asymmetrical septal hypertrophy, pericardial effusion, Wall motion abnormalities, Systolic dysfunction, Diastolic dysfunction - abnormalities were reported The cardiac manifestations of hypothyroidism include sinus bradycardia, pericardial effusion, heart failure and coronary atheroma. Overt hypothyroidism is associated with hyperlipidaemia and coronary artery disease. 2D echocardiography uses the principle of ultrasound reflection of cardiac structures to produce images of the heart. For a transthoracic echocardiogram (TTE), the imaging is performed with a handheld transducer placed directly on the chest wall. In selected patients, a TEE may be performed, in which an ultrasound transducer is mounted on the tip of an endoscope placed in the esophagus and directed toward the cardiac structures. ${ }^{7}$ Study aimed to study various cardiac manifestations in hypo-thyroidism patients

\section{MATERIAL AND METHODS}

A cross sectional study was conducted on 54 cases of hypothyroidism patients, was undertaken to study the cardiac manifestations by ECG and ECHO at tertiary care hospital. Study period was from January 2016 to December 2016. Cardiac symptoms studied were breathlessness, palpitation and chest pain. Sinus bradycardia, Low voltage complex, ST-T changes, Long QT, Ectopics, Axis deviation, Prolonged PR, RBBB were studied by taking repeated ECG.

Diastolic dysfunction, Pericardial effusion, Left ventricular hypokinesia, LVH were studied by doing Echo-cardiography.

Inclusion criteria: All thyroid patients attending medicine OPD.

Exclusion criteria: cardiac patients who has undergone cardiac surgery.

\section{RESULTS}

In the present study which included 54 hypothyroid patients, 40 were females and 14 were males (Table 1). Among cardiovascular symptoms breathlessness was commonest symptom being present in 27 patients followed by palpitation in 17 patients and chest pain in 6 patients (Table 2). Among ECG changes, sinus bradycardia was commonest being present in 20 patients, followed by Low voltage complexes in 11 patients, ST-T changes in 10 patients, Long QT in 6 patients, Ectopics in 3 patients, Axis deviation in 2 patients, Prolonged PR in 1 patient, RBBB in 1 patient(Table 3). Diastolic dysfunction seen in 17 patients, pericardial effusion $(25$ cases $)$ being commonest echocardiographic changes in hypothyroid patients followed by, Left ventricular hypokinesia in 8 patients, LVH in 4 patients (Table 4).

\section{DISCUSSION}

The present study included 54 hypothyroid patients, 40 were females and 14 were males (Table 1). Al-Farttoosi et al. in their study found breathlessness in $75 \%$ of patients, which 
was seen in $37 \%$ of patients in the present study. Angina in their study was present in $11.1 \%$ of patients, whereas present study also showed $11.1 \%$. Bradycardia was present in $47.2 \%$ of patients in their study whereas the present study showed $25.9 \%$ of patients. They also found in their study significant difference between diastolic blood pressure, in present study $62.9 \%$ patients had $\mathrm{DBP}>80$. $^{8}$

A1-Farttoosi et al. found in their study that sinus bradycardia was the commonest ECG finding in hypothyroid patients, the same is concluded from this study too. As in Al-Farttoosi et al.58 study, present study also showed next common findings being low voltage complexes, ST-T changes, prolonged QT respectively. ${ }^{8}$

Among cardiovascular symptoms breathlessness was commonest symptom being present in 27 patients followed by palpitation in 17 patients and chest pain in 6 patients (Table 2). Rawat B et al. ${ }^{9}$ study showed relatively increased thickness of Left ventricle which was found in $7.4 \%$ of patients in present study. Pericardial effusion was present in $32.5 \%$ patients in Hardisty et al. study whereas Rawat B et al. study showed $72 \%$ and Gupta et al. had $45.45 \%$ patients with the same. In this study it was seen in $25.9 \%$ of patients. ${ }^{10}$ Gupta et al. found significant increase in interventricular septum (IVS) and left ventricular posterior wall (LVPW), whereas in our study $7.4 \%$ patients had LVH. ${ }^{10}$

Among ECG changes, sinus bradycardia was commonest being present in 20 patients, followed by Low voltage complexes in 11 patients, ST-T changes in 10 patients, Long QT in 6 patients, Ectopics in 3 patients, Axis deviation in 2 patients, Prolonged PR in 1 patient, RBBB in 1 patient (Table 3). Subclinical hypothyroidism or mild thyroid failure is a common problem, with a prevalence of $3 \%$ to $8 \%$ in the population without known thyroid disease. The prevalence increases with age and is higher in women. After the sixth decade of life, the prevalence in men approaches that of women, with a combined prevalence of $10 \%$. Antithyroid antibodies can be detected in $80 \%$ of patients with $\mathrm{SCH}$, and $80 \%$ of patients with $\mathrm{SCH}$ have a serum TSH of less than $10 \mathrm{mIU} / \mathrm{L} .^{11}$

Subclinical hypothyroidism affects diastolic function and that this abnormality may be reversed by L-T4 substitutive therapy.61 Rodondi et al. in their follow up study found that over 5 years, left ventricular mass increased among those with $\mathrm{TSH} \geq 10.0$, but other echo-cardiographic measurements were unchanged. ${ }^{12}$

Diastolic dysfunction seen in 17 patients, pericardial effusion (25 cases) being commonest echo-cardiographic changes in hypothyroid patients followed by, Left ventricular hypokinesia in 8 patients, LVH in 4 patients (Table 4). Singh et al. describes three cases with varying degrees of atrioventricular block in myxedema, which reverted to normal sinus rhythm following thyroid hormone replacement. ${ }^{13}$

Both phases of heart cycle are early and contemporary involved in subclinical hypothyroidism causing the decrease of intra-myocardial contractility and the impairment of both active and passive phases of diastole. ${ }^{14}$

Hypothyroid cardio-myopathy is an uncommon manifestation of hypothyroidism that can take the form of dilated cardiomyopathy. ${ }^{15}$

\section{CONCLUSION}

The study showcases the significant cardio-vascular changes among the vulnerable age group between 20-40 years. Predominantly females were more affected than males. Hypo-thyrodism patients can have significant cardio-vascular symptoms, breathlessness being the most common symptom followed by palpitation and chest pain. Study of serial ECG changes revealed sinus bradycardia as the commonest finding followed by low voltage complexes, ST-T changes, long QT, ectopics, axis deviation, prolonged PR interval and RBBB. Echo-cardiography changes commonly shows diastolic dysfunction and pericardial effusion. Left ventricular hypokinesia and LVH were other significant findings of echo-cardiography. The above analysis highlights the fact that cardiac evaluation as an important and significant role in management of hypothyroid patients.

\section{REFERENCES}

1. Moolman JA. Thyroid hormone and the heart. Cardiovascular Journal of South Africa 2002;13(4): 159-63.

2. Tang. Low Thyroid Function Leads to Cardiac Atrophy with Chamber Dilatation, Impaired Myocardial Blood Flow, Loss of Arterioles, and Severe Systolic dysfunction. Circulation 2005;112(2):3122-30.

3. Tang. Low Thyroid Function Leads to Cardiac Atrophy Wit $h$ Chamber Dilatation Impaired Myocardial Blood Flow, Loss of Arterioles, and Severe Systolic dysfunction. Circulation 2005; 112(1):3122-30.

4. Forfar. Left ventricular function in hypothyroidism Responses to exercise and beta adrenoceptor blockade. Br Heart J 1982;48(6):278-84.

5. Ansari. Cardiac Complications of Hyperthyroidism: Echocardiographic Evaluation of 69 Hyperthyroid Patients. TAJ 2004;17(5):6-9.

6. Singh. Reversible Atrioventricular Block in Myxedema. Chest 1973;63(1);582-5.

7. Nishimura. Noninvasive Cardiac Imaging: Echocardiography, Nuclear Cardiology, and MRI/CT Imaging. 18th ed. Chapter 229. In: Harrison's Principles of Internal Medicine, Longo, et al., eds. New York: The McGraw- Hill; 2012. pp. 1840-52.

8. Al-Farttoosi. Cardiovascular Manifestations of Primary Hypothyroidism. The Iraqi Postgraduate Medical Journal 2010;9(3):113-9.

9. Rawat. An echocardiographic study of cardiac changes in hypothyroidism and the response to treatment. Kathmandu University Medical Journal 2003;2(1):1827.

10. Gupta. Echocardiographic changes and alterations in lipid profile in cases of subclinical and overt hypothyroidism. The Journal of the Association of Physicians of India 1996;44(4):546,551-3.

11. Ertugrul. Prevalence of Subclinical Hypothyroidism among Patients with Acute Myocardial Infarction. ISRN Endocrinology 2011.

12. Rodondi. Subclinical Thyroid Dysfunction, Cardiac Function and the Risk of Heart Failure: The Cardiovascular Health Study. J Am Coll Cardiol 2008;52(6):1152-9. 
13. Singh. Reversible Atrioventricular Block in Myxedema. Chest 1973;63(5);111-5.

14. Fabrigio. Early textural and functional alterations of left ventricular myocardium in mild hypothyroidism. European Journal of Endocrinology 2006;155(3):3-9.

15. Neito. Asymmetric Septal Hypertrophy Associated with Subclinical Hypothyroidism. Rev Esp Cardiol 2004;57(3):792-6.

Source of Support: Nil; Conflict of Interest: None

Submitted: 22-12-2018; Accepted: 25-12-2019; Published online: 12-02-2019 